\title{
Dissolution and off-stoichiometric formation of compound layers in solid state reactions
}

\author{
Zoltán Erdélyi, Dezső L. Beke, and Andriy Taranovskyy \\ Department of Solid State Physics, University of Debrecen, P.O. Box 2, H-4010 Debrecen, Hungary
}

(Received 18 December 2007; accepted 13 March 2008; published online 2 April 2008)

\begin{abstract}
In many technological processes, intermediate phases are produced by solid state reactions (SSRs) of pure materials initially layered onto each other. It is generally accepted that during heat treatments of such structures, stoichiometric compound films form and then grow, or if such a layer already exists then it growths further. We show that in many realistic cases the compound layer forms and starts to grow highly off stoichiometrically. Moreover, an initially existing stoichiometric compound layer may dissolve then off stoichiometrically reform. Our findings are of primary importance for nanotechnologies where early stages of SSR are utilized; e.g., metallization for silicon-based devices in micro-/nanoelectronics. (C) 2008 American Institute of Physics.
\end{abstract}

[DOI: 10.1063/1.2905334]

It is well known that if one creates a contact between two different, $A$ and $B$, solids and anneals this sandwich structure new intermediate, chemically ordered, $A_{x} B_{y}$ phase(s) can grow. This solid state reaction (SSR) is often utilized in important technological steps. ${ }^{1}$ The process is imagined that due to interdiffusion, an intermixed zone forms and in this-after the diffusion zone became wide enough to allow the formation of the compound layer ${ }^{2,3}$ - the reaction product appears and grows. If the product is a chemically ordered phase, it contains atoms in a well determined, stoichiometric ratio.

This description, however, implicitly assumes that the diffusion profile symmetrically evolves during the formation and growth of an $A B$ (i.e., $A_{0.5} B_{0.5}$ ) reaction product, i.e., as many $A$ atoms enter into the growing intermediate phase as $B$ atoms do; i.e., the $A$ and $B$ atoms have the same mobility in the $A B$ phase. Otherwise the growing phase would not contain $A$ and $B$ atoms in stoichiometric ratio [see Fig. 1(a)].

However, it is hard to imagine that this assumption is valid, since almost in all realistic cases, atoms have very different mobility in different matrices. This is strongly related to the difference of the bonding energies, usually $V_{A A}$ $\neq V_{B B}$. If, for example, $\left|V_{A A}\right|>\left|V_{B B}\right|$, the diffusion of both $A$ and $B$ atoms is much faster in the $B$ than in the $A$ matrix, i.e., atoms can hardly move in pure $A$, but are quite mobile in pure $B$ (diffusion asymmetry). ${ }^{4-6}$ Consequently, at the interface, the $B$ atoms practically cannot penetrate into the $A$ matrix and only the $A$ atoms can dissolve into the $B$ matrix. Furthermore, the dissolved $A$ atoms can diffuse away in the $B$ matrix very fast. This explains the heavy tail distribution of the $A$ atoms and that the interface remains quite abrupt, practically atomic sharp at the 0.5 stoichiometric composition. ${ }^{6}$ Thus, the new $A B$ phase cannot form yet at this stage; it requires the development of an intermixed zone of definite thickness containing $A$ and $B$ atoms in nearly stoichiometric ratio. ${ }^{2,3}$

However, our computer kinetic Monte Carlo (KMC) and kinetic mean field (KMF) calculations (for the details of the used techniques see Refs. 7 and 8, respectively) surprisingly show different behavior: the formation starts already much

\footnotetext{
a) Author to whom correspondence should be addressed. Tel./FAX: +3652 316073 . Electronic mail: zerdelyi@dragon.unideb.hu.
}

earlier than the composition profiles become wide at the expected composition, and the formation happens at offstoichiometric composition [see Fig. 1(b)]. Furthermore, the growing phase is far from being fully ordered at the beginning. Later on, thickening of the compound layer as well as gradual shift of its average composition and degree of order toward the equilibrium value can be observed.

In contrast to the widely accepted classical picture of formation of intermediate phases, in our simulations, we do not observe any "nucleation thickness" for the reaction product: In the symmetric case, the ordered phase immediately starts to grow when the diffusion starts; whereas in the asymmetric case, although the formation of the phase is postponed until the $B$ matrix is filled up to a certain level at which the formation of the ordered layer can already decrease the free energy of the system (kinetic retardation), the interface is still atomically sharp when the new phase starts to form in the $B$ matrix. This is consistent with Hillert's result, ${ }^{9}$ who has observed that for ordering systems (forming ordered structures), there is no theoretical free energy barrier for nucleation in coherent lattices. Obviously, if the formation of the intermediate phase requires, e.g., the rearrangement of the crystalline structure, a positive excess energy can be attributed to the formation of new interfaces and the picture described in Refs. 2 and 3 may be considered.
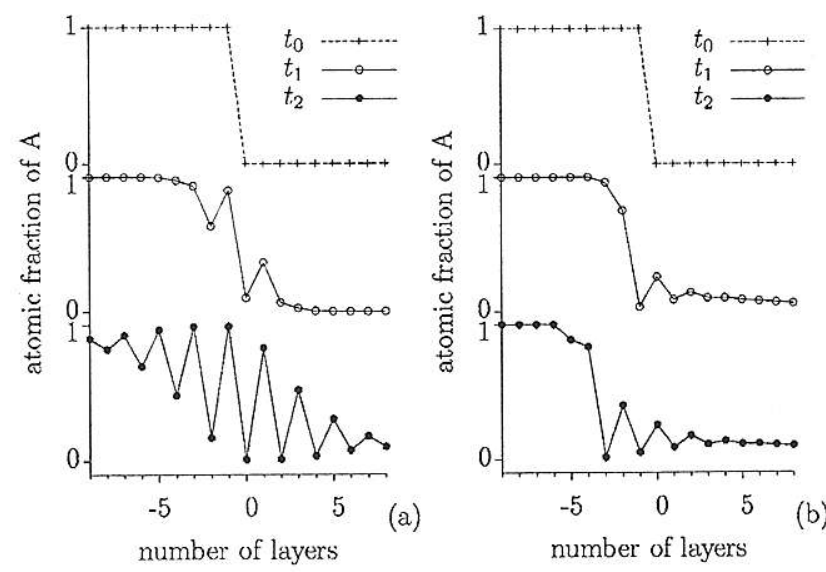

FIG. 1. Time evolution of the composition profile of an $A / B$ diffusion couple during SSR obtained from KMF calculations [bcc(100)]. (a) Symmetric diffusion case: stoichiometric $A B$ intermediate phase formation. (b) Asymmetric diffusion case: off-stoichiometric formation of the new phase. 


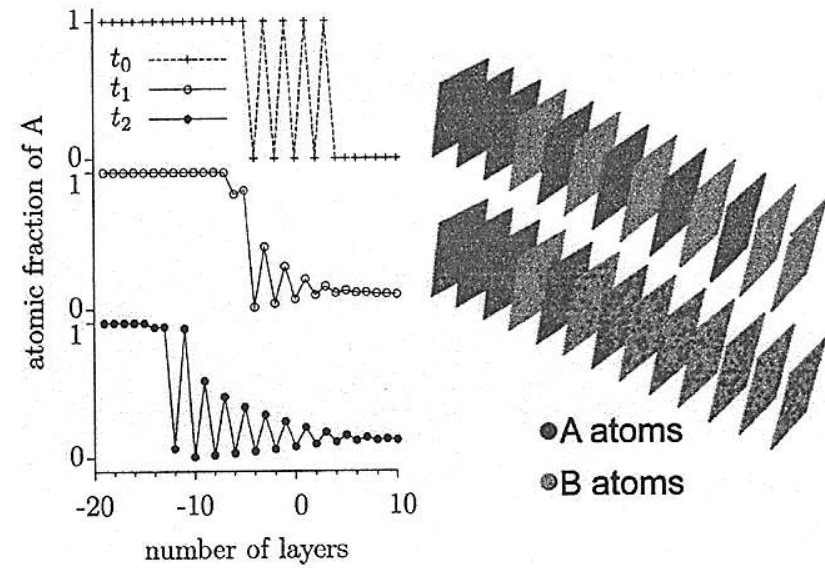

FIG. 2. (Color) Dissolution and off-stoichiometric regrowth of the $A B$ phase in asymmetric diffusion [bcc(100)]. Left panel (KMF): Time evolution of the composition profile. The $A B$ phase dissolves into the $B$ matrix $\left(t_{0}, t_{1}\right)$ and starts to regrow for $t>t_{1}$. However, the order is not uniform $\left(t_{2}\right)$. Right panel (KMC): atomistic view of the initial state and after some time when $A B$ is somewhat dissolved (small part of the KMC cell is shown; lattice planes are detached for better visualization).

Note that the kinetic retardation of the phase formation in the asymmetric case can give an atomistic guideline to interpret the well-known ordered " $\mathrm{Cu}_{3} \mathrm{Au}$ rule" for the growth of different competitive ordered phases: ${ }^{1,10}$ "the first phase to form is always the one where the diffusion is characterized by the lowest activation energy" (i.e., in which the diffusion is the fastest), or in other words, those phase forms first for which the composition is closest to the pure element with the lower melting point.

If there is an ordered stoichiometric phase in the system initially, one usually would expect its further growth. However, KMF and KMC computer simulations show that in the case of asymmetric diffusion, an initial $A B$ phase can be thinned, or even can completely be dissolved (depending on the strength of the diffusion asymmetry, the ordering energy, and the initial thickness of $A B$ ) in the matrix in which the diffusion is faster (matrix $B$; see Fig. 2). The thinning may be in the order of few nanometers in realistic systems, i.e., a few nanometer thick phase can disappear.

This is because for the growth of the $A B$ phase, the number of $A$ atoms entering into this phase at the $A / A B$ interface and the number of $B$ atoms entering at the $A B / B$ interface should be equal to each other. Moreover, since the $A$ and $B$ atoms not only enter into the $A B$ phase but leave it, the outgoing currents should be equal to each other and should be smaller than the incoming currents: $J_{A}^{\text {in }}=J_{B}^{\text {in }}>J_{A}^{\text {out }}=J_{B}^{\text {out }}$ (see Fig. 3). However, for asymmetric diffusion at the beginning, this condition obviously cannot be obeyed, since in this case $J_{A}^{\text {in }}<J_{A}^{\text {out }}$ and $J_{B}^{\text {in }}>J_{B}^{\text {out }}$; i.e., the total amount of $A$ atoms decreases ( $B$ increases) in the $A B$ phase. To keep the stoichiometry, the system will decrease the thickness of the $A B$ phase. Ad absurdum, the system can dissolve the whole $A B$ phase. However, although during the process all the currents decrease, $J_{A}^{\text {out }}$ does it faster than $J_{A}^{\text {in }}$. At a certain point $J_{A}^{\text {out }}$ equals $J_{A}^{\text {in }}$. From here $J_{A}^{\text {out }}<J_{A}^{\text {in }}$, i.e., more $A$ atoms enter into the intermediate phase than leave it; consequently, the $A B$ phase starts to be enriched in $A$ and to regrow, or if the phase has previously disappeared, to "renucleate" off stoichiometrically and to grow (see the right bottom panel in Fig. 3). Of course, also $J_{B}^{\text {out }}<J_{B}^{\text {in }}$, but due to the diffusion asymmetry $J_{B}^{\text {in }}+J_{B}^{\text {out }}>J_{A}^{\text {in }}+J_{A}^{\text {out }}$; thus, the order is not uniform in the $A B$
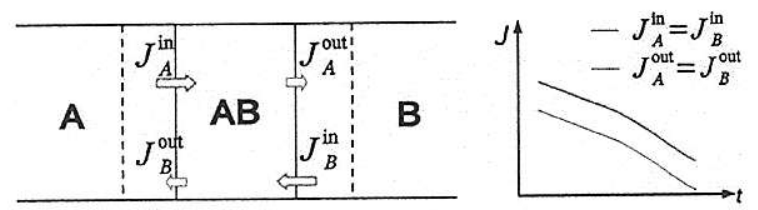

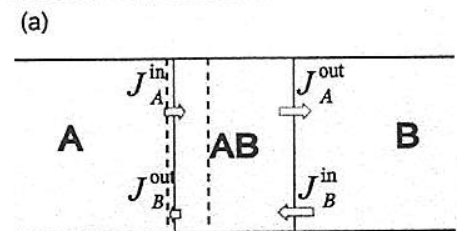

(b)

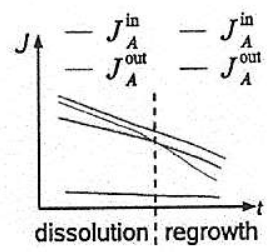

FIG. 3. (Color) Schemes of the incoming and outgoing currents in SSR for (a) symmetric and (b) asymmetric diffusion. (a) Stoichiometric phase growth (dashed line shows the thicker phase) $J_{A}^{\text {in }}=J_{B}^{\text {in }}>J_{A}^{\text {out }}=J_{B}^{\text {out }}$. (b) First $J_{A}^{\text {out }}>J_{A}^{\text {in }}$ and $J_{B}^{\text {in }}>J_{B}^{\text {out }}$, as a result, the $A B$ phase enriches in $B$. To keep the stoichiometry, the phase is thinning (dashed line). All the currents decrease in time, but $J_{A}^{\text {out }}$ faster than $J_{A}^{\text {in, }}$, thus once $J_{A}^{\text {out }}=J_{A}^{\text {in }}$, then $J_{A}^{\text {out }}<J_{A}^{\text {in }}$, i.e., the phase enriches in $A$ and starts to grow.

phase. Closer to the $A B / B$ interface the phase is more off stoichiometric [see the profile $t_{2}$ in Figs. 1(b) and 2].

We note that we have repeated the above calculations starting with a homogeneous and completely disordered $A_{0.5} B_{0.5}$ "phase" between the pure $A$ and $B$ matrices, and also with a completely disordered but linearly diffused interface (constant composition gradient at the interface). We have found similar comportment as previously, i.e., the decrease of the "intermediate phase" followed by off-stoichiometric formation and growth of the (new) ordered phase.

Moreover, we also note that the dissolution and offstoichiometric formation/growth of the phase can also be observed in multilayers. In this case, the minimal thickness which can be reached by the $A B$ phase in dissolution also depends on the initial thickness of the matrix in which the diffusion is faster.

In this paper, we have shown that an intermediate $A B$ ordered phase off stoichiometrically forms and the order is not uniform in the growing phase in the case of asymmetric diffusion. In our simulations, we do not observe any "nucleation thickness" for the reaction product. Moreover, an initial intermediate $A B$ ordered (or even completely disordered) phase may dissolve and later on off stoichiometrically reform. Our findings are of primary importance for nanotechnologies in which early stages of SSRs are utilized and since they take place on the few nanometer scale, they can be attained by recent experimental techniques.

This work was supported by the OTKA Board of Hungary (Nos. K67969 and K61253). One of the authors of this paper is a grantee of the "Bolyai János" scholarship.

${ }^{1}$ F. M.d'Heurle, J. Mater. Res. 3, 167 (1988).

${ }^{2}$ P. J. Desre and A. R. Yvari, Phys. Rev. Lett. 64, 1533 (1990).

${ }^{3}$ A. M. Gusak, Ukr. Phys. J. 35, 725 (1990) [in Russian].

${ }^{4}$ Z. Erdélyi, I. A. Szabó, and D. L. Beke, Phys. Rev. Lett. 89/16, 165901 (2002)

${ }^{5}$ Z. Erdélyi, M. Sladecek, L.-M. Stadler, I. Zizak, G. A. Langer, M. KisVarga, D. L. Beke, and B. Sepiol, Science 306, 1913 (2004).

${ }^{6}$ D. L. Beke and Z. Erdélyi, Phys. Rev. B 73, 035426 (2006).

${ }^{7}$ For example, Z. Erdélyi and D. L. Beke, Phys. Rev. B 70, 245428 (2004).

${ }^{8}$ For example, F. Soisson, A. Barbu, and G. Martin, Acta Mater. 44, 3789 (1996).

${ }^{9}$ M. Hillert, Acta Metall. 9, 525 (1961).

${ }^{10}$ F. M.d'Heurle, P. Gas, and J. Philbert, Defect Diffus. Forum 143-147, 529 (1997) 